\title{
Percepción de estudiantes de veterinaria acerca del uso de la eutanasia en animales de compañía en Lima, Perú
}

\author{
Perception of veterinary students about the use of euthanasia in companion \\ animals in Lima, Peru
}

\author{
Alexandra Rizo-Patrón ${ }^{1,4}$, Daphne León ${ }^{1}$, Patricia Herrera² ${ }^{2}$ Pablo Silva ${ }^{3}$, \\ Néstor Falcón ${ }^{1}$
}

\section{Resumen}

\begin{abstract}
El objetivo del estudio fue conocer la percepción de estudiantes de veterinaria de tres universidades de Lima, Perú, acerca del uso de la eutanasia en animales de compañía. Para ello se validó un instrumento de recolección de información que incluyó variables demográficas del encuestado, preguntas acerca del uso de eufemismos y aceptación de la eutanasia en diferentes escenarios y proposiciones para la valoración ética de la eutanasia. Se recolectaron 640 encuestas, correspondiendo el $65.5 \%$ a estudiantes mujeres. Todos los estudiantes conocían el término eutanasia, siendo el eufemismo «dormir» el más utilizado como estudiante $(63.3 \%)$ y el término eutanasia, propiamente dicho, el que utilizarían profesionalmente (45.5\%). La eutanasia fue aceptada principalmente en caso de enfermedad crónica, tanto cuando se trataba de su propio animal $(83.3 \%)$ como en su futura actuación profesional (83.1\%). El 68.6\% de estudiantes mencionó haber participado en al menos una eutanasia de animales de compañía. La valoración de la eutanasia
\end{abstract}

${ }^{1}$ Laboratorio de Epidemiología y Salud Pública en Veterinaria, Facultad de Medicina Veterinaria y Zootecnia, Universidad Peruana Cayetano Heredia, Lima, Perú

${ }^{2}$ Oficina de Tutoría Académica y Consejería Psicológica, Facultad de Medicina Veterinaria y Zootecnia, Universidad Peruana Cayetano Heredia, Lima, Perú

${ }^{3}$ Laboratorio de Fisiología y Farmacología, Facultad de Medicina Veterinaria y Zootecnia, Universidad Peruana Cayetano Heredia, Lima, Perú

${ }^{4}$ E-mail: alexandra.rizo.patron.m@upch.pe

Recibido: 17 de enero de 2021

Aceptado para publicación: 16 de julio de 2021

Publicado: 24 de agosto de 2021

CLos autores. Este artículo es publicado por la Rev Inv Vet Perú de la Facultad de Medicina Veterinaria, Universidad Nacional Mayor de San Marcos. Este es un artículo de acceso abierto, distribuido bajo los términos de la licencia Creative Commons Atribución 4.0 Internacional (CC BY 4.0) [https:// creativecommons.org/licenses/by/4.0/deed.es] que permite el uso, distribución y reproducción en cualquier medio, siempre que la obra original sea debidamente citada de su fuente original 
como «muerte digna» tuvo elevada aceptación (63.7\%), así como el rechazo mayoritario a la idea de que la eutanasia «devalúa la profesión médico veterinaria» $(73.3 \%)$. El sufrimiento animal (87.9\%) y la calidad de vida de estos $(62.7 \%)$ fueron las principales consideraciones para decidir la eutanasia. Se concluye que la percepción de los estudiantes de veterinaria hacia la eutanasia en animales de compañía fue favorable, por lo que se requiere reforzar la formación profesional en aspectos médicos, legales y éticos que permitan su adecuada recomendación y aplicación.

Palabras clave: eutanasia, muerte, veterinaria, mascotas

\section{Abstract}

The aim of this study was to determine the perception of veterinary students from three universities in Lima, Peru, about the use of euthanasia in companion animals. For this, an information collection instrument was validated that included demographic variables of the respondent, questions about the use of euphemisms and acceptance of euthanasia in different settings, and proposals for the ethical assessment of euthanasia. In total, 640 surveys were collected, $65.5 \%$ corresponding to female students. All the students knew the term euthanasia, the euphemism «sleeping» being the most used as a student $(63.3 \%)$ and the term euthanasia, properly speaking, the one they would use professionally (45.5\%). Euthanasia was accepted mainly in case of chronic disease, both when it was about their own animal $(83.3 \%)$ and in their future professional performance $(83.1 \%)$. Besides, $68.6 \%$ of students mentioned having participated in at least one euthanasia of companion animals. The assessment of euthanasia as a «dignified death» had high acceptance (63.7\%), as well as the majority rejection of the idea that euthanasia «devalues the veterinary medical profession» (73.3\%). Animal suffering (87.9\%) and their quality of life $(62.7 \%)$ were the main considerations in deciding euthanasia. It is concluded that the perception of veterinary students towards euthanasia in companion animals was favourable, so it is necessary to reinforce professional training in medical, legal and ethical aspects that allow its adequate recommendation and application.

Key words: euthanasia, death, veterinary, pets

\section{INTRODUCCIÓN}

La tenencia de animales de compañía ha favorecido el desarrollo de un fuerte vínculo humano-animal (Anderson, 2008), que los ha llevado a ser considerados como miembros de la familia (Sable, 2013). Además, la tenencia de estos animales ha producido un impacto positivo en las personas, favoreciendo la conservación de la salud, el bienestar psicosocial e inclusive, la recuperación de condiciones de salud graves (Walsh, 2009; Antonacopoulos y Pychyl, 2010); sin embar- go, la expectativa de vida de los animales de compañía es menor que la del ser humano, por lo que la posibilidad de experimentar la muerte de ellos se convierte en evento inevitable para sus propietarios (Cartolin et al., 2019).

Entre los desenlaces de la vida de un animal se encuentra la eutanasia. Este evento involucra factores previos y el método utilizado, el cual debe ser realizado sin dolor, mínimo o libre de miedo y estrés, confiable, reproducible, irreversible, sencillo, seguro y rápido de realizar (Cabrejo, 2016). Debe de 
ser llevado a cabo por personal capacitado para garantizar el bienestar animal y debe ser aplicado ante la presencia de una enfermedad dolorosa, incurable o vejez (AVMA, 2020).

La eutanasia en animales se clasifica en activa y pasiva. La eutanasia activa corresponde a la aplicación de alguna sustancia que genere un estado de inconsciencia seguido de un paro cardiorrespiratorio, que termina con la vida del animal; con pleno consentimiento del propietario (Ríos-González et al., 2017). En este caso, es importante la selección del medicamento eutanásico debido a que de este depende si el animal sufrirá o no (Melgar et al., 2015). En el caso de la eutanasia pasiva, se deja de aplicar el tratamiento, medicamentos o cualquier procedimiento necesario para prolongar la vida del paciente, siendo este el tipo de eutanasia menos usado en medicina veterinaria (RíosGonzález et al., 2017).

Dado que la eutanasia es un procedimiento que involucra tanto a médicos veterinarios, pacientes y propietarios, es necesario conocer qué tan preparados se encuentran emocional, ética y metodológicamente los estudiantes de medicina veterinaria para su realización; así como conocer los criterios para la toma de la decisión y su correcto desempeño empático al momento de presentarla como opción al propietario. En este contexto, el objetivo del estudio fue conocer la percepción de los estudiantes de medicina veterinaria acerca del uso de la eutanasia en animales de compañía en tres universidades de la ciudad de Lima, Perú.

\section{Materiales y MéTOdos}

La recolección de información se realizó en facultades de ciencias veterinarias de tres universidades ubicadas en la ciudad de Lima, Perú. La elaboración de la base de datos y los análisis estadísticos se realizaron en el Laboratorio de Epidemiología y Salud Pública de la Facultad de Medicina Veterina- ria y Zootecnia de la Universidad Peruana Cayetano Heredia. La investigación correspondió a un estudio observacional descriptivo de corte transversal.

La población objetivo estuvo constituida por la totalidad de estudiantes matriculados en el periodo académico 2019-II. El tamaño de muestra se calculó mediante la fórmula de tamaño de muestra para estimar una proporción para poblaciones desconocidas, considerando una proporción referencial de $50 \%$ para la variable participación en un acto de eutanasia (valor aleatorio cuando no se conoce un valor referencial), un nivel de confianza del $95 \%$ y un error máximo admisible del 5\% (Seoane et al., 2007). El tamaño de muestra calculado fue de 385 encuestas como mínimo, las que se trató de distribuir en forma proporcional en cada institución involucrada en el estudio.

El instrumento de recolección de información consistió en un cuestionario estructurado de preguntas con alternativas múltiples, de una o más opciones de respuesta de pregunta precodificadas (Sánchez, 2007), el que fue validado por juicio de expertos, recibiendo revisiones sucesivas hasta que se alcanzó la aceptación unánime de ellos. El cuestionario fue diseñado para auto aplicación e incluyó una sección de datos generales (edad, sexo y año de estudio del encuestado) y una sección de preguntas específicas: idea acerca del término eutanasia; términos escuchados para referirse a la eutanasia y el que utilizaría como profesional; situaciones en las que aceptaría la eutanasia en un animal de su propiedad y en el futuro como profesional; haber tocado el tema de eutanasia en algún curso; participación en una eutanasia; percepción de la eutanasia como asesinato, acto antiético y muerte digna; percepción de la eutanasia como acto que devalúa la profesión médico veterinaria; del sufrimiento del animal como factor importante para realizar la eutanasia; del respeto a la vida del animal hasta su deceso; de la calidad de vida del animal como factor importante relacionado a 
la eutanasia; y aceptación como válida de la definición de la eutanasia.

La aplicación de la encuesta fue coordinada con las autoridades de las facultades involucradas. La encuesta se realizó en las aulas de clase bajo la supervisión del docente responsable de la clase y el investigador responsable del estudio, quien ofreció las indicaciones para la autoadministración del cuestionario y resolvió las dudas que se presentaron durante su aplicación. Los encuestados tuvieron un tiempo promedio de 10 minutos para la resolución del cuestionario.

La base de datos se generó en Microsoft Office Excel 2019 y después fue analizada en el programa estadístico Stata 15.0. Las variables del estudio fueron resumidas mediante estadística descriptiva. Se utilizó la media y desviación estándar para resumir la variable cuantitativa edad. Las variables cualitativas se resumieron mediante frecuencias absolutas y relativas. La asociación de las características demográficas y los resultados a la encuesta se evaluaron mediante la prueba de Chi Cuadrado por tablas de contingencia y en el caso de confrontar las respuestas por efecto de escenarios (actual como estudiante o futuro profesional), la significación del cambio de opinión fue evaluada mediante la prueba de Chi Cuadrado de McNemar. En todos los casos se trabajó con un nivel de significancia del $5 \%$.

El estudio se desarrolló con la aprobación del Comité Institucional de Ética de la Universidad Peruana Cayetano Heredia (Constancia 624-28-19). La participación en el estudio fue voluntaria y el manejo de los cuestionarios fue anónimo.

\section{Resultados}

Se entrevistó a 640 estudiantes de veterinaria de los cuales $60.8 \%$ (389) estudiaba en la Universidad «A», 26.4\% (169) en la Universidad «B» y $12.8 \%$ (82) en la Univer- sidad «C». El 34.5\% (221) eran varones y 65.5\% (419) mujeres. La distribución de los estudiantes según el año que cursaban fue la siguiente: $19.7 \%$ (126) del primer año, $20.0 \%$ (128) del segundo, 22.7\% (145) del tercero, $19.8 \%$ (127) del cuarto y $17.8 \%$ (114) del quinto.

Todos los encuestados mencionaron haber escuchado el término eutanasia. El término «dormir» fue el elegido con mayor frecuencia como el escuchado al referirse a dicha práctica como estudiante y el de eutanasia, propiamente dicho, el que utilizarían como profesional. La distribución de la frecuencia de uso de diversos términos para referirse a la eutanasia se describe en el Cuadro 1.

La principal razón por la cual los estudiantes aceptarían la eutanasia para su propio animal, así como en su futuro profesional, sería en caso de enfermedades crónicas, sin que la opinión haya cambiado por efecto del escenario propuesto. También se mantuvieron similares proporciones para ambos escenarios como el caso de avanzada edad y comportamiento agresivo. Sin embargo, hubo un aumento significativo para el escenario de actuación profesional al evaluar la aceptación de la eutanasia en caso de brotes de enfermedad y viaje/mudanza del propietario (Cuadro 2).

El 29.2\% (187) de los encuestados mencionaron haber recibido información acerca de eutanasia en algún curso a lo largo de sus estudios universitarios. Múltiples cursos, entre generales, formativos y especializados, fueron mencionados sin mostrar una frecuencia mayoritaria por alguno de ellos en los ciclos de estudio y universidades del estudio. El 60.3\% (76/126) de estudiantes del primer año, el 66.4\% (85/128) del segundo, el 62.8\% (91/145) del tercero, el 77.2\% (98/127) del cuarto y el 78.1\% (89/114) del quinto mencionaron haber participado en una eutanasia. Solo se encontró diferencia estadística entre alumnos del primer y quinto año $(\mathrm{p}<0.05)$. Así mismo, 66.1\% (146/221) de los estudiantes 
Cuadro 1. Frecuencia de términos utilizados para referirse a la eutanasia en condición de estudiante de medicina veterinaria y los que utilizarían como profesional (noviembre-diciembre, 2019; $\mathrm{n}=640$ )

\begin{tabular}{lccccc}
\hline Términos referidos a & \multicolumn{2}{c}{ Escuchado $^{1}$} & \multicolumn{2}{c}{ El que utilizaría ${ }^{1}$} & \multirow{2}{*}{ Sig. } \\
\cline { 2 - 5 } eutanasia & $\mathrm{n}$ & $\%$ & $\mathrm{n}$ & $\%$ & \\
\hline Eutanasia & 259 & 40.5 & 291 & 45.5 & 0.017 \\
Dormir & 405 & 63.3 & 236 & 36.9 & 0.000 \\
Hacer descansar & 306 & 47.8 & 207 & 32.3 & 0.000 \\
Sacrificar & 298 & 46.6 & 145 & 22.7 & 0.000 \\
Matar & 99 & 15.5 & 18 & 2.8 & 0.000 \\
\hline
\end{tabular}

${ }^{1}$ Los estudiantes encuestados podían ofrecer más de una respuesta

Cuadro 2. Situaciones en las que estudiantes de medicina veterinaria encuestados aceptarían la eutanasia en su propio animal y como futuro profesional (noviembre-diciembre, 2019; $\mathrm{n}=640$ )

\begin{tabular}{lccccc}
\hline \multirow{2}{*}{ Situación evaluada } & \multicolumn{2}{c}{$\begin{array}{c}\text { Con su propio } \\
\text { animal }^{1}\end{array}$} & \multicolumn{2}{c}{$\begin{array}{c}\text { Como } \\
\text { profesional }^{1}\end{array}$} & \multirow{2}{*}{ Sig. } \\
\cline { 2 - 5 } & $\mathrm{n}$ & $\%$ & $\mathrm{n}$ & $\%$ & \\
\hline Enfermedad crónica del animal & 533 & 83.3 & 532 & 83.1 & 1.000 \\
Brote de enfermedad & 316 & 49.4 & 378 & 59.1 & 0.000 \\
Avanzada edad del animal & 229 & 35.8 & 217 & 33.9 & 0.235 \\
Comportamiento agresivo & 123 & 19.2 & 135 & 21.1 & 0.188 \\
Viaje/mudanza del propietario & 43 & 6.7 & 60 & 9.4 & 0.009 \\
\hline
\end{tabular}

${ }^{1}$ Los estudiantes encuestados podían ofrecer más de una respuesta

varones y el 70.1\% (293/418) mujeres habrían participado de una eutanasia, no encontrándose diferencia por género.

Los estudiantes mostraron su desacuerdo acerca de la valoración de la eutanasia con los términos «asesinato» y «antiético», pero una elevada aceptación a valorar la eutanasia como «muerte digna». Las propor- ciones de estas respuestas tendieron a ser mayores en las mujeres, en comparación a los hombres.

En cuanto a los enunciados propuestos, se rechazó mayoritariamente la idea de que «la eutanasia devalúa a la profesión veterinaria», pero recibieron apoyo mayoritario los enunciados que indicaron que «el sufrimiento 
Cuadro 3. Percepción acerca de la valoración de la eutanasia y enunciados propuestos por parte de estudiantes de medicina veterinaria de tres universidades de Lima, Perú (noviembre-diciembre, 2019

\begin{tabular}{lcccccc}
\hline Valoración y enunciado respecto a & $\begin{array}{c}\text { En desacuerdo y } \\
\text { totalmente en } \\
\text { eutanasia }\end{array}$ & \multicolumn{2}{c}{$\begin{array}{c}\text { Indiferente o } \\
\text { neutral }\end{array}$} & $\begin{array}{c}\text { De acuerdo y } \\
\text { totalmente de } \\
\text { acuerdo }\end{array}$ \\
\cline { 2 - 8 } & $\mathrm{n}$ & $\%$ & $\mathrm{n}$ & $\%$ & $\mathrm{n}$ & $\%$ \\
\hline $\begin{array}{l}\text { Valoración } \\
\quad \text { Asesinato }\end{array}$ & 430 & 67.2 & 87 & 13.6 & 123 & 19.2 \\
$\quad \begin{array}{l}\text { Antiético } \\
\quad \begin{array}{l}\text { Muerte digna } \\
\text { Enunciados }\end{array}\end{array}$ & 438 & 68.4 & 140 & 21.9 & 62 & 9.7 \\
$\quad \begin{array}{l}\text { Eutanasia devalúa a la profesión } \\
\text { médico veterinario }\end{array}$ & 469 & 15.9 & 130 & 20.3 & 408 & 63.8 \\
$\quad \begin{array}{l}\text { Considera sufrimiento del paciente } \\
\text { al decidir la eutanasia }\end{array}$ & 44 & 6.9 & 34 & 5.3 & 562 & 87.8 \\
$\quad \begin{array}{l}\text { La vida de un animal debe de ser } \\
\text { respetada hasta su fin }\end{array}$ & 135 & 21.1 & 120 & 18.8 & 385 & 60.2 \\
$\quad \begin{array}{l}\text { Es preferible la muerte del animal a } \\
\text { mantenerlo sin calidad de vida }\end{array}$ & 141 & 22.0 & 98 & 15.3 & 401 & 62.7 \\
\hline
\end{tabular}

se debe de considerar para tomar la decisión de hacer la eutanasia a un animal», «que la vida del animal se debe de respetar hasta el final» y que «es preferible la eutanasia a mantener un animal sin calidad de vida». En estas percepciones, las proporciones tendieron a ser mayores en las mujeres, en comparación a los hombres. La valoración de la eutanasia y a los enunciados propuestos a los estudiantes se presenta en el Cuadro 3. El total de encuestados tuvo una definición aceptable al término eutanasia.

\section{Discusión}

El estudio encuentra una mayor participación de estudiantes de los primeros años de estudio, a diferencia de los últimos años, quienes suelen encontrarse en prácticas fi- nales en espacios de docencia extrauniversitario. Asimismo, la participación femenina predominó en concordancia al aumento de este grupo dentro de la educación superior (Díaz, 2008), que también se refleja en la carrera de Medicina Veterinaria (Paredes y Zuazo, 2014).

Los resultados muestran que los eufemismos más utilizados por los estudiantes en la actualidad, «dormir»y «hacer descansar», difieren de los términos eufemísticos utilizados en la década de los 90 que eran «destruir», «acabar»y «adormecer» (CCAC, 1998). Este resultado muestra un mayor respeto por el vínculo humano-animal de compañía y exige además responsabilidad, respeto y un buen actuar de parte del profesional veterinario, más allá de la obligación ética que tiene de proporcionar al animal una eutanasia adecuada (AVMA, 2020). 
El referirse a la eutanasia con eufemismos busca alejar el rechazo a escuchar la palabra muerte (Marín, 2018) y, en esta parte, los resultados del estudio coinciden con una investigación realizada en Guayaquil, Ecuador, en estudiantes de medicina veterinaria, de otras carreras y público en general, en donde la aceptación de la eutanasia en diversos escenarios y situaciones fue mayor al utilizar el término «dormir» (León y Plúas, 2019). Los estudiantes deben de tener presente que la eutanasia se considera un evento importante dentro de la rutina del médico veterinario, por lo que las condiciones como la manejen podrían generar conflictos en el bienestar propio, del animal de compañía y de los propietarios (Degeling et al., 2017).

Los estudiantes de veterinaria muestran su aceptación a la eutanasia en animales propios, así como en su condición profesional futura, principalmente para casos de enfermedad crónica del paciente. Ello resulta adecuado teniendo en consideración que una avanzada edad del animal y la ocurrencia de neoplasias se encuentran dentro de los causales más comunes para el ejercicio de eutanasia en caninos (Bonnett et al., 2005; Fighera et al., 2008). A esto se suma las enfermedades degenerativas de gravedad e incurables, la nula respuesta a los tratamientos, y un pronóstico de vida inferior a seis meses, entre otros, que son motivos para realizar procedimientos adicionales de carácter paliativo que concluyen cuando la calidad de vida del paciente empieza a deteriorarse, tomando así una decisión como la eutanasia que se muestra como un evento final de la vida para el animal (Cabrejo, 2016).

El uso de la eutanasia para situaciones de brotes de enfermedad de implicancia epidemiológica y de salud pública como la rabia tuvo menor aceptación. En este sentido se debe tener en cuenta que la Norma Técnica de Salud para la Prevención y Control de la Rabia Humana en el Perú menciona que la eutanasia de tipo selectiva debe de incluir animales que han sido mordidos o han estado involucrados en un área foco y/o se encuentren en condición de vagabundos por la zona del brote de rabia, ya que ellos representan un riesgo para la salud pública (MINSA, 2017). Sin embargo, estos criterios están siendo cuestionados en los últimos años y se sugieren otras alternativas que no tengan tanto impacto, sobre todo en los propietarios, cuyos canes se encuentren involucrados en la epidemiología de la enfermedad (Cleaveland et al., 2014; Castillo-Neyra et al., 2016).

La Ley $\mathrm{N}^{\circ} 27596$, publicada en 2001, también considera la eutanasia para casos de agresión por parte de canes clasificados como potencialmente peligroso, cuando estos ocasionan daños físicos graves o muerte de personas y/o animales, cuando han participado en peleas de canes clandestina y cuando hayan sido recogidos por la municipalidad y en un plazo de 30 días no han sido retirados por algún propietario $\mathrm{y} / \mathrm{o}$ ha sido imposible reincorporarlos en la sociedad. Se excluye la aplicación de esta parte de la norma en casos que los canes hayan actuado en defensa propia, de su propietario, de un tercero o para la integridad de la propiedad privada.

La aceptación estudiantil del uso de la eutanasia en un animal con comportamiento agresivo debe poner en consideración la necesidad de introducir cursos dirigidos hacia la etología y bienestar animal, con la finalidad de comprender el lenguaje y el posterior manejo que requiere cada animal (Uribe et al., 2007). Dado que las mordeduras de caninos y felinos son un problema de salud pública, es necesario que el médico veterinario informe a los propietarios sobre aspectos del desarrollo comportamental normal de los animales de compañía para evitar el desarrollo de conductas agresivas (Palacio et al., 2005). Además, se conoce que la agresividad en perros y gatos constituye una de las principales razones para su posterior abandono, incluso en condición física determinada como saludable (Heath, 2007). Por ello, los médicos veterinarios deben de generar una influencia directa en la relación propietario-animal de compañía, recomendándoles estrategias 
adecuadas para prevenir o tratar algún problema de comportamiento en sus animales (Patronek y Dodman, 1999; Landsberg et al., 2003).

Aproximadamente un tercio de los estudiantes mencionó haber revisado el tema de eutanasia en algún curso a lo largo de sus estudios universitarios. Esta proporción se considera baja, comparado con los observados en el plan de estudios de estudiantes de veterinaria de Australia y Nueva Zelanda (Littlewood et al., 2018). Es responsabilidad de los centros formadores brindar espacios de discusión y desarrollar estrategias de enseñanza lo más próximo al ejercicio real de los médicos veterinarios, para así establecer la capacidad de discernimiento sobre método adecuado de eutanasia, medicamentos y escenarios a los que se han de exponer en la labor profesional. Ello debe de conducir a la búsqueda del equilibro entre el esfuerzo de curar y el de velar por la calidad de vida de un animal de compañía y el de la familia propietaria (Cabrejo, 2016). Por otra parte, es también esencial que los estudiantes, en su rol como futuros profesionales, reciban el conocimiento adecuado que les permita decidir cuándo intervenir o cuando detener algún tipo de procedimiento dentro del tratamiento de un animal de compañía (Meza et al., 2011).

La alta participación de los estudiantes en procedimientos eutanásicos muestra que durante su formación profesional han experimentado vivencialmente la metodología básica del procedimiento, mientras que la relación con el factor año de estudio, indica que son los últimos años en donde se cursa temas de especialidad, donde los estudiantes tienen mayor oportunidad de presenciar una eutanasia. Estos resultados se condicen con los encontrados por las universidades de Australia y Nueva Zelanda, donde los estudiantes llevan los cursos relacionados a la eutanasia en los últimos años de la carrera (Littlewood et al., 2018).
El estudio reportó un mayoritario rechazo de los estudiantes a la idea de que la eutanasia en animales de compañía causa una devaluación de la profesión como médicos veterinarios, puesto que esta práctica es un procedimiento en donde se muestra la misión de la medicina veterinaria, cual es la de proporcionar calidad de vida a los animales. Por ello, es necesario que los egresados de la carrera conozcan cómo manejar estas y otras situaciones de carácter emocionalmente significativo, tanto para ellos mismos como para los propietarios y los mismos animales de compañía (Meza et al., 2011; Littlewood et al., 2018).

El factor sufrimiento también se encuentra considerado dentro de la toma de decisión del procedimiento eutanásico para los estudiantes encuestados, como medio que busque aliviar ese sufrimiento (Ríos-González et al., 2017). En este tema, el dolor es independiente en cada animal y responde a variables importantes como estado de atención y estado emocional, por lo que es indispensable que los estudiantes reciban una educación adecuada al grado de dificultad que significa la evaluación del dolor en un determinado paciente (Aigé y Cruz, 2001).

El respetar la vida del animal de compañía hasta el final y la preferencia de la eutanasia por encima de una pobre calidad de vida del paciente, también fueron hallazgos positivos del estudio, lo que pudo ser favorecidos debido a que dentro del campo de la medicina veterinaria, existen un conjunto de responsabilidades éticas que involucran temas de bienestar animal, ética, legislación, formas de docencia y también reconocimiento científico y una base fisiológica para el dolor, lo que hace capaces a los médicos veterinarios de valorar dicho dolor en sus pacientes y la recomendación de la eutanasia como medio para aliviarla (Gutiérrez y Calvo, 2011).

Con relación al género del estudiante y la percepción favorable hacia la eutanasia, los resultados fueron variados. El estudio 
encontró una tendencia a que la frecuencia de encuestados con percepción favorable a la eutanasia sea por parte de las mujeres. Similar resultado se encontró en un estudio realizado en Holanda con estudiantes de medicina (Muller et al., 1996). Sin embargo, estudios realizados en estudiantes y residentes de medicina humana mostraron una mayor percepción favorable hacia la eutanasia en los varones que en las mujeres, tanto en Puerto Rico (Ramírez-Rivera et al., 2006) como en Sudan (Ahmed y Kheir, 2006). Por ello, establecer una regularidad asociada al sexo es difícil porque habría que profundizar el análisis con relación al sexo y otros factores no controlados en algunos estudios (sociales, económicos, políticos, religiosos, etc.). Ello ha de ser aún más difícil en el caso de estudiantes de veterinaria, donde los estudios en este tema son aún incipientes.

Se debe de considerar que la eutanasia en animales de compañía representa un procedimiento que busca ofrecer una última alternativa a los pacientes, por lo tanto, dicho acto clínico debe de ser realizado por personal capacitado, el cual garantice siempre el bienestar animal, con una muerte sin dolor ni angustia, ni para el animal, propietario y el profesional que lo ejecuta.

El estudio concluye que la percepción de los estudiantes de veterinaria hacia la eutanasia en animales de compañía fue favorable; por ello, se requiere una formación profesional integral en el tema, dado que la decisión de aplicarla no solo ha de pasar por una valoración médica del estado de salud del animal, sino que además debe considerar aspectos legales y éticos que el Médico Veterinario debe de tomar en cuenta al discernir cuando ello es aceptable. Además, debe estar preparado para el procedimiento en sí, como el correcto manejo empático con el dueño, lo que ha de tener un impacto beneficioso para el animal, el propietario y el propio Médico Veterinario.

\section{Literatura Citada}

1. Ahmed A, Kheir M.. 2006. Attitudes towards euthanasia among final-year Khartoum University medical students. Eastern Mediterranean Health J 12: 391-397.

2. Aigé V, Cruz I. 2001. El dolor en los pequeños animales: bases neuroanatómicas, reconocimiento y tratamiento. Consulta Difus Vet 9(78): 63-70.

3. Anderson DC. 2008. The humancompanion animal bond. Ref Libr 41: 7-23.

4. Antonacopoulos NMD, Pychyl TA. 2010. An examination of the potential role of pet ownership, human social support and pet attachment in the psychological health of individuals living alone. Anthrozoös 23: 37-54. doi: 10.2752/175303710X12627079939143

5. [AVMA] American Veterinary Medical Association. 2020. Guidelines for the euthanasia of animals. 2020 ed. Illinois: American Veterinary Medical Association. $121 \mathrm{p}$.

6. Bonnett BN, Egenvall A, Hedhammar A, Olson P. 2005. Mortality in over 350,000 insured Swedish dogs from 1995 2000: I. Breed-, gender-, age- and cause-specific rates. Acta Vet Scand 46: 105-120. doi: 10.1186/1751-0147-46-105

7. Cabrejo C. 2016. La eutanasia en medicina veterinaria de pequeños animales. REDVET 17(7). [Internet]. Disponible en: http://www.veterinaria.org/revistas/ redvet/n070716.html

8. Cartolin X, Herrera AP, León D, Falcón N. 2019. Del vínculo humano con los animales de compañía al impacto emocional por su pérdida. Rev Inv Vet Perú 35: e17837. doi: 10.15381/ rivep.v31i2.17837

9. Castillo-Neyra R, Levy MZ, Náquira C. 2016. Efecto del sacrificio de perros vagabundos en el control de la rabia canina. Rev Perú Med Exp Salud Pública 33: 772-779. doi: 10.17843/rpmesp.2016.334.2564 
10. [CCAC] Canadian Council on Animal Care. 1998. Guidelines. [Internet]. Available in: https://www.ccac.ca/en/ standards/guidelines/generalguidelines.html

11. Cleaveland S, Beyer H, Hampson K, Haydon D, Lankester F, Lembo T, et al. 2014. The changing landscape of rabies epidemiology and control. Onderstepoort J Vet Res 81(2). doi: 10.4102/ ojvr.v81i2.731

12. Degeling C, Fawcett A, Collins T, Hazel S, Johnson J, Lloyd J, et al. 2017. Students' opinions on welfare and ethics issues for companion animals in Australian and New Zealand veterinary schools. Aust Vet J 95: 189-193. doi: 10.1111/avj.12590

13. Díaz, JJ. 2008. Educación superior en el Perú: tendencias de la demanda y la oferta. En: Benavides M (ed). Análisis de programas, procesos y resultados educativos en el Perú: contribuciones empíricas para el debate. Lima: GRADE. p 83-129.

14. Fighera RA, Souza TM, Silva MC, Brum JS, Graça DL, Kommers GD, et al. 2008. Causas de morte e razões para eutanásia de cães da Mesorregião do Centro Ocidental rio-Grandense (1965-2004). Pesq Vet Bras 28: 223-230.

15. Gutiérrez E, Calvo P. 2011. La formación del médico veterinario: una invitación a la ética. Rev Univ de La Salle 54: 161-173.

16. Heath SE. 2007. The welfare of cats. In: Behaviour problems and welfare. Dordrecht: Rochlitz I. p 91-118.

17. Landsberg G, Hunthausen W, Ackerman L. 2003. Feline aggression. In: Handbook of behavior problems of dog and cat. Philadelphia: Elsevier.

18. León SL, Plúas PD. 2019. Uso del término «dormir» en referencia a la práctica de eutanasia en mascotas. Tesis de Médico Veterinario. Ecuador: Univ. de Guayaquil. 61 p.
19. Ley $N^{\circ} 27596$. Ley que regula el régimen jurídico de canes. En: Separata de Normas Legales del Diario Oficial «El Peruano». Lima: 2001.

20. Littlewood KE, Beausoleil NJ, Stafford KJ, Stephens C, Collins T, Fawcett A, et al. 2018. Exploring how end-of-life management is taught to Australasian veterinary students. Part 1: technical euthanasia. Vet Rec 183: 691. doi: 10.1136/vr.104775

21. Marín F. 2018. La eutanasia: un derecho del siglo XXI. Gac Sanit 32: 381-382.

22. Melgar MJ, Pérez M, Camiña M, Puerta JL, Cantalapiedra JJ, Yllera MM, et al. 2015. Bienestar animal: métodos de eutanasia y aturdimiento. Santiago de Compostela: Junta de Galicia Ministerio de Medio Rural y Mar. 94 p.

23. Meza JL, Garavito L, Rodríguez JA, Solarte R, Torres $N E$, Venegas $S$, et al. 2011. La muerte: siete visiones, una realidad. Bogotá: Ed Pontificia Universidad Javeriana. $216 \mathrm{p}$.

24. [MINSA] Ministerio de Salud. 2017. Norma técnica de salud para la vigilancia, prevención y control de rabia humana en el Perú. Perú. [Internet]. Disponible en: https://busquedas.elperuano.pe/ normaslegales/aprueban-norma-tecnicade-salud-para-la-vigilancia-prevenciresolucion-ministerial-no-0242017minsa-1475513-1/

25. Muller MT, Onwuteaka-Philipsen BD, Kriegsman DMW, van der Wal G. 1996. Voluntary active euthanasia and doctor-assisted suicide: knowledge and attitudes of Dutch medical students. Med Educ 20: 428-433. doi: 10.1111/j.13652923.1996.tb00863.x

26. Palacio J, León M, García-Belenguer S. 2005. Aspectos epidemiológicos de las mordeduras caninas. Gac Sanit 19: 50-58.

27. Paredes A, Zuazo J. 2014. Características de los ingresantes a la carrera de Medicina Veterinaria y Zootecnia de la Universidad Peruana Cayetano Heredia en el periodo 2010-2013. Salud Tecnol Vet 2: 113-119. 
28. Patronek GJ, Dodman NH. 1999. Attitudes, procedures, and delivery of behavior services by veterinarians in small animal practice. J Am Vet Med Assoc 215: 1606-1611.

29. Ramírez-Rivera J, Cruz J, JaumeAnselmi F. 2006. Euthanasia, assisted suicide and end-of-life care: attitudes of students, residents and attending physicians. P R Health Sci J 25: 325-329.

30. Ríos-González CM, De Benedictis-Serrano GA, Córdova-Rivas GJ, Contreras-Romero ML, ContrerasLugo LV. 2017. Conocimiento y percepción sobre eutanasia en estudiantes de medicina de diecisiete países latinoamericanos. Mem Inst Investig Cienc Salud 16: 58-65.

31. Sable P. 2013. The pet connection: an attachment perspective. Clin Social Work J 41: 93-99.
32. Sánchez E. 2007. Capítulo 6: Diseño de cuestionarios. En: Medicina de Familia 33: 412-416.

33. Seoane, T., Martín-Sánchez, E., Martín, J.L., Lurueña-Segovia, S., Alonso, F.J. 2007. Capítulo 5: Selección de la muestra: técnicas de muestreo y tamaño muestral, SEMERGEN - Medicina de Familia. 33(9):466-471. doi: 10.1016/ S1138-3593(07)73931-X

34. Uribe MT, Lozano I, Calderón NA. 2007. Importancia de la etología en la formación de los estudiantes de Medicina Veterinaria y de Zootecnia en la Universidad de La Salle «reflexiones pedagógicas y aportes bibliográficos. Rev Med Vet 13: 95-105.

35. Walsh F. 2009. Human-animal bonds I: The relational significance of companion animals. Fam Process 48: 462-480. doi: 10.1111/j.1545-5300.2009.01296.x 